\title{
Biochemical Analysis of Interaction between Kringle Domains of Plasminogen and Prion Proteins with Q167R Mutation
}

\author{
Jeongmin Lee ${ }^{1 \dagger}$, Byoung Woo Lee ${ }^{2 \dagger}$, Hae-Eun Kang ${ }^{3}$, Kevine K. Choe ${ }^{4}$, Moosik Kwon ${ }^{2}$, and Chongsuk Ryou ${ }^{4 *}$ \\ ${ }^{1}$ Division of Zoonoses, Center for Immunology and Pathology, National Institute of Health, Korea Centers for Disease Control and \\ Prevention, Cheongwon 28159, Republic of Korea \\ ${ }^{2}$ Department of Genetic Engineering, Sungkyunkwan University, Suwon 16419, Republic of Korea \\ ${ }^{3}$ Division of Foreign Animal Disease, Animal and Plant Quarantine Agency, Gimcheon 39660, Republic of Korea \\ ${ }^{4}$ Department of Pharmacy and Institute of Pharmaceutical Science and Technology, Hanyang University, Ansan 15588, Republic of Korea
}

Received: February 13, 2017

Revised: March 3, 2017

Accepted: March 8, 2017

First published online

March 9, 2017

*Corresponding author

Phone: +82-31-400-5811;

Fax: +82-31-400-5958;

E-mail: cryou2@hanyang.ac.kr

${ }^{\dagger}$ These authors contributed equally to this work.

pISSN 1017-7825, eISSN 1738-8872

Copyright(C) 2017 by

The Korean Society for Microbiology and Biotechnology
The conformational change of cellular prion protein $\left(\mathrm{PrP}^{\mathrm{C}}\right)$ to its misfolded counterpart, termed $\operatorname{PrP}^{\mathrm{Sc}}$, is mediated by a hypothesized cellular cofactor. This cofactor is believed to interact directly with certain amino acid residues of $\operatorname{PrP}^{\mathrm{C}}$. When these are mutated into cationic amino acid residues, $\mathrm{PrP}^{\mathrm{Sc}}$ formation and prion replication halt in a dominant negative (DN) manner, presumably due to strong binding of the cofactor to mutated $\operatorname{PrP}^{\mathrm{C}}$, designated as DN PrP mutants. Previous studies demonstrated that plasminogen and its kringle domains bind to $\operatorname{PrP}$ and accelerate $\operatorname{PrP}^{\mathrm{Sc}}$ generation. In this study, in vitro binding analysis of kringle domains of plasminogen to Q167R DN mutant PrP (PrPQ167R) was performed in parallel with the wild type (WT) and Q218K DN mutant PrP (PrPQ218K). The binding affinity of PrPQ167R was higher than that of WT PrP, but lower than that of PrPQ218K. Scatchard analysis further indicated that, like PrPQ218K and WT PrP, PrPQ167R interaction with plasminogen occurred at multiple sites, suggesting cooperativity in this interaction. Competitive binding analysis using L-lysine or L-arginine confirmed the increase of the specificity and binding affinity of the interaction as PrP acquired DN mutations. Circular dichroism spectroscopy demonstrated that the recombinant PrPs used in this study retained the $\alpha$-helix-rich structure. The $\alpha$-helix unfolding study revealed similar conformational stability for WT and DN-mutated PrPs. This study provides an additional piece of biochemical evidence concerning the interaction of plasminogen with DN mutant PrPs.

Keywords: Prion protein, dominant negative mutant, cofactor, plasminogen

\section{Introduction}

The cellular prion protein $\left(\operatorname{PrP}^{\mathrm{C}}\right)$ most abundantly expressed in neuronal cells of the central nervous system is a glycosylated protein tethered to the plasma membrane via a glycosylphosphatidylinositol anchor [1]. Conformational conversion of $\mathrm{PrP}^{\mathrm{C}}$ to its disease-associated isoform, scrapie prion protein $\left(\operatorname{PrP}^{S c}\right)$, is an important biochemical event for prion propagation [2] because the proteinaceous pathogens causing transmissible spongiform encephalopathy in humans and animals, termed prions, are composed of $\operatorname{PrP}^{\mathrm{Sc}}$ [1]. Thus, formation of $\mathrm{PrP}^{\mathrm{Sc}}$ facilitated by conformational conversion of $\operatorname{PrP}^{\mathrm{C}}$ to $\mathrm{PrP}^{\mathrm{Sc}}$ infers propagation of prions.

A number of investigations, including molecular biological studies of $\mathrm{PrP}^{S c}$ formation in cultured cells, in vitro structural studies of $\operatorname{PrP}^{C}$, and prion transmission studies using transgenic mice, have suggested that a cellular cofactor is involved in the conversion of $\operatorname{PrP}^{\mathrm{C}}$ to $\operatorname{PrP}^{\mathrm{Sc}}[3-8]$. It is proposed that this as-yet unidentified cofactor directly interacts with prion protein $(\operatorname{PrP})$ and increases $\operatorname{PrP}^{\mathrm{Sc}}$ formation. Several hypothetical properties concerning the interaction of PrP with cofactors and the consequence of their interaction in $\mathrm{PrP}^{\mathrm{Sc}}$ propagation have been proposed (Summarized in [9]). Mechanistically, the cellular cofactor 

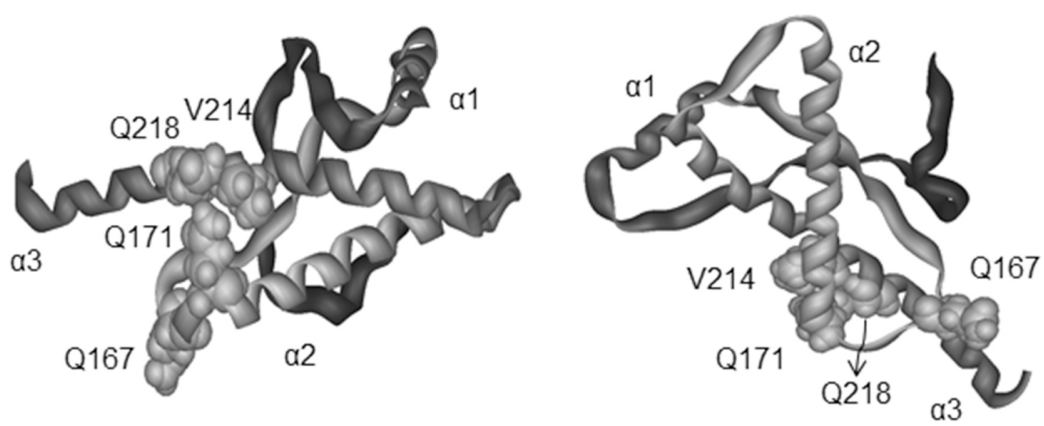

Fig. 1. Four amino acid residues of prion protein $(\operatorname{PrP})$ proposed to interact with the hypothesized cofactor.

The functional groups of these residues are depicted as space-filling spheres on the $\beta 2-\alpha 2$ loop and the $\alpha 3$ helix. Spatial coordination of these PrP residues may create the site for interaction with the hypothesized cofactor. Two different rotational views of mouse PrP (PDB ID: 2L1H) with the identical conformation are presented.

is hypothesized to bind to $\operatorname{Pr} \mathrm{P}^{\mathrm{C}}$, and alters $\operatorname{Pr} \mathrm{P}^{\mathrm{C}}$ into $\operatorname{PrP}$ with a transient conformation that interacts with $\operatorname{PrP}^{\mathrm{Sc}^{\mathrm{C}}}$ for conversion [10]. For generation of $\mathrm{PrP}^{\mathrm{Sc}}$, the cofactor is proposed to bind to Q167, Q171, V214, and Q218 in the $\beta 2$ $\alpha 2$ loop (164-174) and C-terminus (215-223) of $\operatorname{PrP}^{\mathrm{C}}$ to perform an auxiliary function for conformational conversion of $\operatorname{PrP}^{\mathrm{C}}$ into $\operatorname{PrP}^{\mathrm{Sc}}$ (Fig. 1) [6, 11]. When $\operatorname{PrP}^{\mathrm{C}}$ mutated at these sites into basic amino acid residues, such as Q167R and Q218K, was overexpressed in the cells and animals, $\mathrm{PrP}^{\mathrm{Sc}}$ formation and prion replication were prevented in prion-infected hosts in a dominant negative (DN) manner, presumably due to high-affinity binding of the cofactor to $\operatorname{PrP}^{\mathrm{C}}$ with DN mutations $[8,11]$.

Investigations to generate infectivity or transmissibility of synthetic prions demonstrated that non-proteinaceous macromolecules, such as lipids, function as cofactors for prion replication [12]. Nevertheless, their molecular interaction with $\mathrm{PrP}$ is imprecisely described in biochemical terms. Independent of these approaches, additional attempts to search for protein cofactors that interact physically with the PrP molecule have revealed a number of proteins that bind directly to or associate indirectly with them [13]. The biochemical interaction of $\operatorname{PrP}$ with these putative protein cofactors is partly described, whereas the role of most putative protein cofactors in PrP conversion has not been clearly elucidated.

Among many putative protein cofactors, however, plasminogen was found to stimulate $\mathrm{PrP}^{\mathrm{Sc}}$ propagation [14] and bind directly to the PrP molecule $[15,16]$. In functional studies, conversion of $\operatorname{PrP}^{C}$ to $\operatorname{PrP}^{S c}$ in the cell-free system and cultured cells is enhanced by plasminogen and its kringle domains [14]. Studies for biochemical binding analysis reported that plasminogen specifically pulls down $\mathrm{PrP}^{\mathrm{SC}}$ of the tissue homogenate from prion-infected humans and animals in affinity capture assays [15, 17, 18], and kringle domains of plasminogen are also identified to interact with recombinant PrPQ218K during panning experiments using a mouse brain phage display expression cDNA library [16]. The latter demonstrated that the binding affinity of PrPQ218K to kringle domains is higher than that of wild type (WT) PrP, which correlates with the phenomenon that $\operatorname{PrP}^{\mathrm{C}}$ with DN mutations are less susceptible to be converted into $\operatorname{PrP}^{\mathrm{Sc}_{\mathrm{C}}}$ than WT $\operatorname{PrP}^{\mathrm{C}}[11,19]$.

Despite a partial correspondence between plasminogen and the hypothetical cofactor in functional and biochemical binding perspectives, it is still premature to conclude that plasminogen is synonymous with the cofactor of $\operatorname{PrP}$ conversion. Thus, it is necessary to further investigate whether plasminogen and the hypothetical cofactor share common properties. Although several studies on the interaction between plasminogen and $\mathrm{PrP}$ were previously conducted [15-18, 20], a few unresolved or incompletely addressed issues still remain, such as PrP binding affinity difference when PrP interacts with DN PrP mutants. In this study, the biochemical effect of Q167R DN mutation on PrP itself and on the binding affinity of PrPQ167R to the kringle domain of plasminogen was compared with that of the DN mutation Q218K and WT PrP, which were previously reported.

\section{Materials and Methods}

\section{Gene Cloning and Site-Directed Mutagenesis}

The PCR-amplified gene fragment for mouse PrP, encoding from the 23rd to 231st amino acid residues (PrPWT), was cloned into the NdeI and BamHI sites of $\mathrm{pET} 23 \mathrm{~b}$ plasmid vector (Novagen, EMDMillipore, USA) by the method described in a previous report [21]. Forward (5'-AAA AAG CGG CCA AAG 
CCT-3') and reverse (5'-GGA TCT TCT CCC GTC GTA ATA-3') primers were used for PCR amplification. The resultant plasmid, termed pET23b-PrPWT, was used for site-directed mutagenesis by the method of splicing-by-overlap extension [22], using PCR primers and mutagenesis oligonucleotides for Q167R (forward oligo: 5'- GAT CGC TAC AGC AAC CAG AAC-3'; reverse oligo: 5'-GTA GCG ATC CAC TGG CCT-3') and for Q218K (forward oligo: 5'- TAC AAG AAG GAG TCC CAG-3'; reverse oligo: 5'CTT CTT GTA CTG GGT GAC G-3') substitutions. The PCR primers and mutagenesis oligonucleotides were purchased from CosmoGenetech (Korea). The mutagenized PCR products were also cloned into the NdeI and BamHI sites of pET23b plasmid vector. The DNA cyclic amplification profile was a total of 30 cycles composed of denaturation at $95^{\circ} \mathrm{C}$ for $30 \mathrm{sec}$, annealing at $55^{\circ} \mathrm{C}$ for $30 \mathrm{sec}$, and extension at $72^{\circ} \mathrm{C}$ for $30 \mathrm{sec}$. The resultant recombinant plasmids, pET23b-PrPQ167R, pET23b-PrPQ218K, and pET23b-PrPWT, were used to confirm the site-directed mutations by DNA sequence determination using T7 forward primers (Macrogen, Korea). For verification of PrP gene subcloning in pET23b, recombinant plasmids were digested with $\mathrm{XhoI}$ and analyzed by $0.8 \%$ agarose gel electrophoresis.

\section{Production of Recombinant PrP}

E. coli BL21 (DE3) (Invitrogen, USA) was transformed with pET23b-PrPQ167R, pET23b-PrPQ218K, or pET23b-PrPWT. The His-tagged recombinant WT and mutated PrPs (PrPWT, PrPQ167R, and PrPQ218K) were expressed by induction with $1 \mathrm{mM}$ isopropyl $\beta$-D-1-thiogalactopyranoside (IPTG) for $6 \mathrm{~h}$. Cells were harvested and suspended in $10 \mathrm{ml}$ of $50 \mathrm{mM}$ Tris- $\mathrm{HCl}, \mathrm{pH}$ 8.0. The cells were sonicated after freezing-and-thawing five times and then centrifuged at $12,000 \times g$ and $4^{\circ} \mathrm{C}$ for $30 \mathrm{~min}$. The insoluble fraction was washed with washing buffer I (100 mM Tris- $\mathrm{HCl}, \mathrm{pH}$ 8.0) followed by washing buffer II (100 mM Tris-HCl, pH 8.0, 0.5\% Tergitol (SigmaAldrich, USA), $1 \mathrm{M} \mathrm{NaCl}$ ). Inclusion bodies were solubilized in unfolding buffer (100 mM Tris- $\mathrm{HCl}, \mathrm{pH}$ 8.0, $6 \mathrm{M}$ guanidine- $\mathrm{HCl}$ ) with brief sonication and shaking. The supernatant recovered by centrifugation was loaded onto a HiTrap Chelating HP column (GE Healthcare Bio-Sciences, Sweden). Purification was performed according to the manufacturer's instructions. After incubation at $4^{\circ} \mathrm{C}$ for $12 \mathrm{~h}$, the column was washed three times using a salt buffer (20 mM Tris- $\mathrm{HCl}, \mathrm{pH} 8.0,5 \mathrm{mM}$ imidazole, $500 \mathrm{mM} \mathrm{NaCl}$ ). Recombinant PrPs were recovered using an elution buffer $(20 \mathrm{mM}$ Tris- $\mathrm{HCl}$, pH 7.4, $500 \mathrm{mM}$ imidazole, $50 \mathrm{mM} \mathrm{NaCl}$ ).

\section{Refolding of Recombinant PrP}

To obtain the $\alpha$-helix-rich form of $\operatorname{PrP}$, the purified recombinant $\operatorname{PrP}$ was refolded by a process modified from a previous report [23]. The protein was suspended in refolding buffer (100 mM Tris$\mathrm{HCl}$, pH 7.4, $100 \mathrm{mM}$ glycine, $1 \mathrm{M}$ urea, $100 \mathrm{mM}$ oxidized glutathione, $1 \mathrm{mM}$ reduced glutathione) and then centrifuged at $12,000 \times g$ and $4^{\circ} \mathrm{C}$ for $10 \mathrm{~min}$. The supernatant was dialyzed twice against a large volume (100-fold) of dialysis solution ( $20 \mathrm{mM}$ sodium acetate, $\mathrm{pH} 5.5,0.005 \%(\mathrm{w} / \mathrm{v})$ sodium azide) at $4^{\circ} \mathrm{C}$ overnight.

\section{Circular Dichroism (CD) Spectroscopy}

Far-UV CD spectra and thermal transitions were measured using a JASCO-810 CD spectrometer (JASCO, USA) with $1 \mathrm{~nm}$ bandwidth [24]. Recombinant $\operatorname{PrP}(3.5-6.5 \mu \mathrm{M})$ in $20 \mathrm{mM}$ sodium acetate ( $\mathrm{pH}$ 5.5) was used for the $\mathrm{CD}$ analysis. The $\mathrm{CD}$ data were expressed in mean residue ellipticity, $[\theta]_{\mathrm{MRW}}\left(\mathrm{deg} \cdot \mathrm{cm}^{2} / \mathrm{dmol}\right)$. Thermal transition profiles of each recombinant $\operatorname{PrP}$ were measured at $222 \mathrm{~nm}$ under temperature conditions of $37-90^{\circ} \mathrm{C}$. The data were normalized and corrected for the pre- and post-transitional baselines.

\section{SDS-Polyacrylamide Gel Electrophoresis and Immunoblotting}

Recombinant PrPs were analyzed by SDS-PAGE and western blotting. SDS-PAGE was performed in $12 \%$ gels and the protein bands were visualized by Coomassie blue staining. The detailed procedure for western blotting was described elsewhere [25] Primary anti-PrP 6H4 antibody (1:5,000; Prionics, Switzerland) and secondary goat anti-mouse IgG antibody conjugated with peroxidase (1:5,000; Sigma-Aldrich) were used in this study.

\section{Protein-Protein Binding Assays}

To analyze the interaction of WT and DN mutant PrPs with plasminogen, a modified protocol for capture ELISA was performed according to Ryou et al. [16]. Briefly, $10 \mathrm{ng}$ of kringle domains of human plasminogen, designated $\mathrm{K}(1+2+3)$ lysine-binding site I (Sigma-Aldrich), was immobilized onto a 96-well ELISA plate and then incubated with increasing concentrations $\left(1 \times 10^{-1} \sim 1 \times 10^{-6} \mathrm{nM}\right)$ of PrPWT, PrPQ167R, and PrPQ218K. The bound recombinant $\operatorname{PrP}$ was detected by using goat polyclonal anti-PrP serum (1:5,000: gifted from Dr. H-J Woo, Seoul National University, Korea) and rabbit anti-goat IgG antibody conjugated with alkaline phosphatase (Abcam, UK). The Pierce pNPP ( $p$-nitrophenyl phosphate; Sigma-Aldrich), a colorimetric soluble substrate of alkaline phosphatase, was used for detection of absorbance at 405 nm using the SpectraMax microplate reader (Molecular Devices, USA).

Binding activity of WT and DN mutant PrPs to kringle domains was further analyzed with the method of Scatchard [26]. Binding affinity was measured using the term of dissociation constant $\left(K_{\mathrm{d}}\right)$ The number of binding sites was also determined on the basis of Scatchard plots.

For binding competition studies, 1-10 mM L-lysine, L-arginine, L-alanine, or L-aspartic acid (Sigma-Aldrich) was added prior to binding of kringle domains to recombinant WT and DN mutant PrPs. Other assay steps were identical to those described above.

All assays were repeated at least three times, independently. The results are expressed as the mean \pm standard deviation. Statistical analysis was performed using the Student $t$-test.

\section{Results}

\section{Cloning of WT and DN Mutant PrP Genes}

To obtain recombinant PrPs, molecular cloning of the WT 
A

167 th residue

Mouse PrP gene Reference GAT CAGTACAGC. pET23b-PIPWT GATCAGTACAGC. pET23b-PIPQ167R GATCGOTACAGC pET23b-PIPQ218K GATCAGTACAGC.

$$
C A G \rightarrow Q
$$

$\mathrm{CGC} \rightarrow \mathrm{R}$ 218th residue

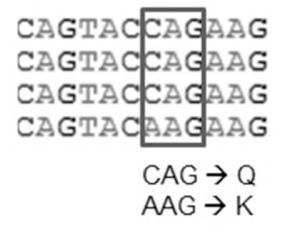

B

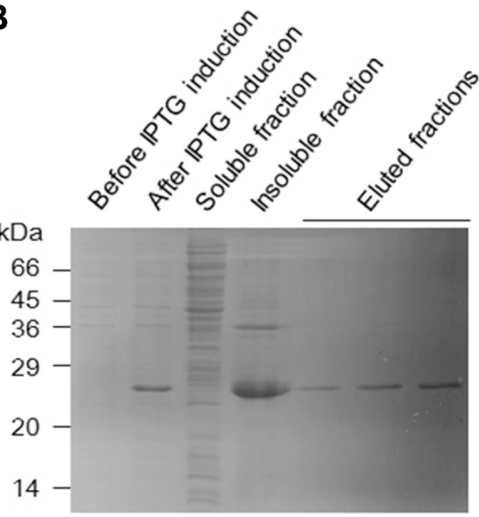

PrPWT

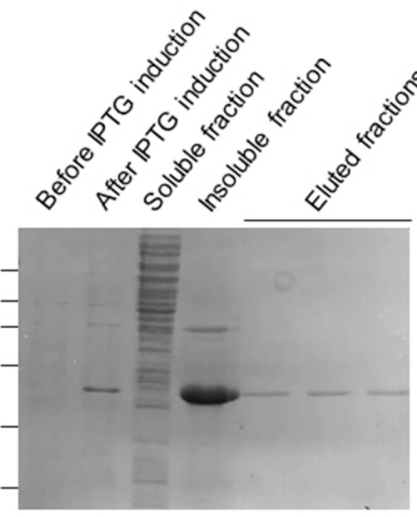

PrPQ167R

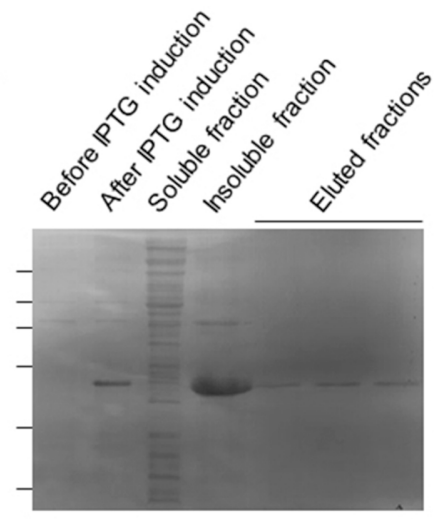

PrPQ218K

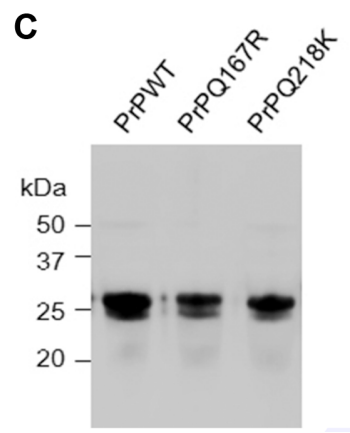

Fig. 2. Expression and purification of recombinant wild-type (WT) and dominant negative (DN) mutant prion proteins (PrPs). (A) DNA sequence determination of WT and DN mutant PrP genes to specify mutations. Sequencing data were aligned to the mouse PrP gene (NCBI NM_001278256.1). (B) Coomassie blue staining of SDS-PAGE gel for recombinant PrPWT, PrPQ167R, and PrPQ218K using the fractions obtained during expression and purification. (C) Western blotting of purified PrPs using anti-prion antibody (clone 6H4).

and DN mutant $\operatorname{PrP}$ genes was performed. The DNA fragment for the WT PrP gene was PCR-amplified and cloned into the NdeI and BamHI sites of bacterial expression plasmid pET23b $(3.665 \mathrm{~kb})$. The DNA fragment for the WT PrP gene was also used as a template for spliced-by-overlap extension to generate site-directed mutations of Q167R and Q218K. Mutated PrP gene fragments were cloned by the same strategy described above. To check the insertion of WT and DN mutant PrP DNA fragments $(0.627 \mathrm{~kb})$ in the vector, the recombinant plasmids were digested with XhoI, which cuts the plasmid only once at the downstream of the insertion site. The linearized recombinant plasmids migrated between the 4 and $5 \mathrm{~kb}$ DNA markers, corresponding to the expected band size of $4.292 \mathrm{~kb}(3.665 \mathrm{~kb}+0.627 \mathrm{~kb})$ (data not shown). This was further confirmed by PCR amplification of the insert DNA fragment, which migrated between the 0.6 and $0.7 \mathrm{~kb}$ DNA markers (data not shown). DNA sequencing of the inserts verified altered nucleotide sequences for Q167R and Q218K (Fig. 2A).

\section{Expression and Purification of Recombinant WT and DN Mutant PrPs}

The recombinant plasmids were used to express the Histagged WT and DN mutant PrPs in bacteria. The pET-based expression system drove a high level of PrP expression controlled by IPTG induction, and accumulated $\operatorname{PrP}$ in a form of insoluble aggregates in inclusion bodies (Fig. 2B). 

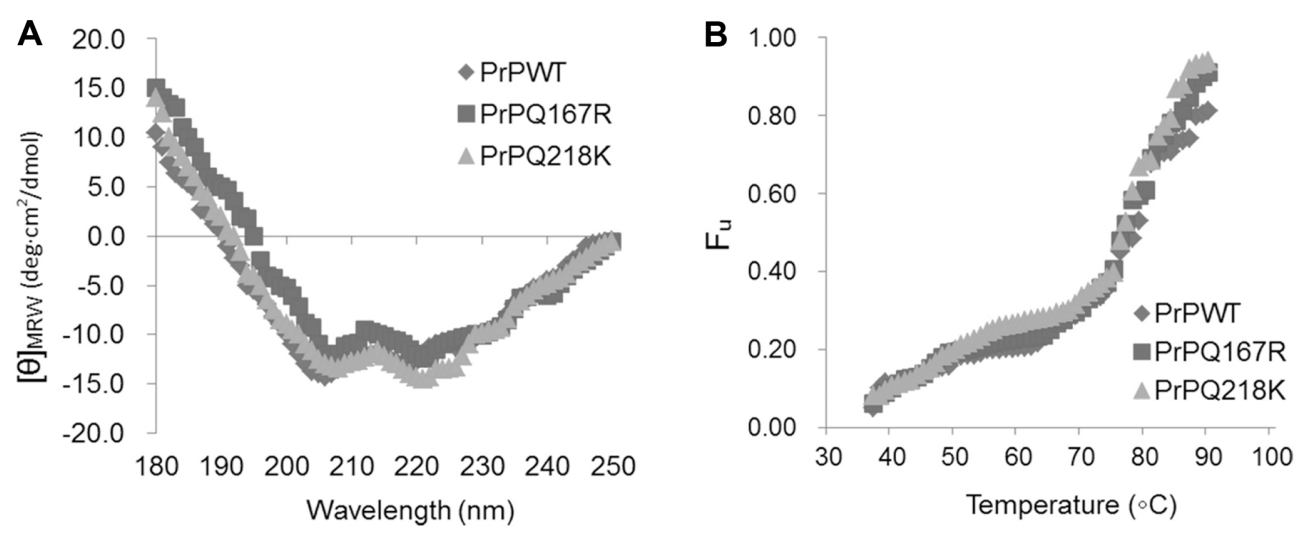

Fig. 3. Secondary structure and thermal stability of wild-type (WT) and dominant negative (DN) mutant prion proteins (PrPs). (A) Far-UV CD spectra of PrPWT, PrPQ167R, and PrPQ218K. [0] $]_{\mathrm{MRW}}$, mean residue ellipticity $\left(\mathrm{deg} \cdot \mathrm{cm}^{2} / \mathrm{dmol}\right)$. (B) Normalized thermal unfolding transitions of PrPWT, PrPQ167R, and PrPQ218K measured at $222 \mathrm{~nm}$. $\mathrm{F}_{\mathrm{u}}$ fraction of unfolded protein.

The recombinant PrPs (PrPWT, PrPQ167R, PrPQ218K) were purified on the basis of binding affinity between the $6 \times$ His-tag and Ni-chelating medium under a denaturing condition with $6 \mathrm{M}$ guanidine-HCl. The molecular mass of the purified recombinant $\operatorname{PrP}$ was $\sim 25-26 \mathrm{kDa}$, judged by SDS-PAGE and western blot analysis (Fig. 2B and 2C). Owing to the tag and flanking sequences $(\sim 2-3 \mathrm{kDa})$, the His-tagged recombinant PrPs were deduced to be bigger than the intrinsic molecular mass of full-length $\operatorname{PrP}(23 \mathrm{kDa})$.

\section{Conformation Analysis of Recombinant WT and DN Mutant PrPs}

The purified recombinant WT and DN mutant PrPs were subjected to refolding. To determine the conformational states of refolded PrPs for use in binding studies, secondary structure analysis was performed using CD spectroscopy. The CD spectra of refolded PrPs exhibited highly similar $\mathrm{CD}$ spectrometric features and represented the typical $\alpha$-helix-rich conformation, showing two negative bands of comparable magnitude at 222 and $208 \mathrm{~nm}$ and a stronger positive band near $190 \mathrm{~nm}$ (Fig. 3A). This suggests that the effect of DN mutations was negligible for PrP to be folded into the $\alpha$-helix-rich conformation.

To measure the conformational stability of the WT and DN mutant PrPs in the $\alpha$-helix-rich conformation,

Table 1. Dissociation constants $\left(K_{\mathrm{d}}\right)$ for two different binding sites of recombinant prion proteins (PrPs).

\begin{tabular}{ccc}
\hline Recombinant PrP & $K_{\mathrm{d} 1}(\mu \mathrm{M})$ & $K_{\mathrm{d} 2}(\mu \mathrm{M})$ \\
\hline PrPWT & 2.05 & 88.66 \\
PrPQ167R & 1.25 & 12.16 \\
PrPQ218K & 0.16 & 0.41 \\
\hline
\end{tabular}

diminishment of the $\alpha$-helix-specific negative band at $222 \mathrm{~nm}$ was determined during thermal denaturation. Overall, the WT and DN mutant PrPs showed similar patterns for unfolding, showing comparable half-maximal melting temperature $\left(T_{\mathrm{m}}\right)$ at $\sim 78^{\circ} \mathrm{C}$ (Fig. 3B). However, it is notable that PrPWT appeared to have less unfolded fractions than PrPQ167R and PrPQ218K at high temperatures.

\section{Affinity Binding of Recombinant WT and DN Mutant PrPs to Kringle Domains of Plasminogen}

To compare the difference in protein-protein interaction, the binding activity of recombinant WT and DN mutant PrPs to kringle domains of plasminogen was measured. All recombinant PrPs demonstrated binding activity to kringle domains, but with a different scale (Fig. 4A). As the concentration of recombinant PrPs increased in binding to the fixed quantity of kringle domains, the dose-dependent binding activity resulted in sigmoidal curves, suggesting cooperativity during their binding. The binding activity of PrPQ167R was intermediary between those of PrPWT and PrPQ218K. This result also showed that the interactions of kringle domains with DN mutant PrPs were stronger than that with PrPWT. The strength of interaction was also supported by Scatchard analysis of the binding activity. The sigmoidal binding activity was transformed not into a single straight line with a slope, but into two bent lines with different slopes (Fig. 4B), suggesting that two binding sites exist for the interaction. Calculation of the dissociation constants for individual binding sites of each interaction (Table 1) revealed that the binding affinity of the PrPQ167R-kringle domain interaction was greater than that of the PrPWT-kringle domain interaction, but less that that of the PrPQ218K-kringle domain interaction. 

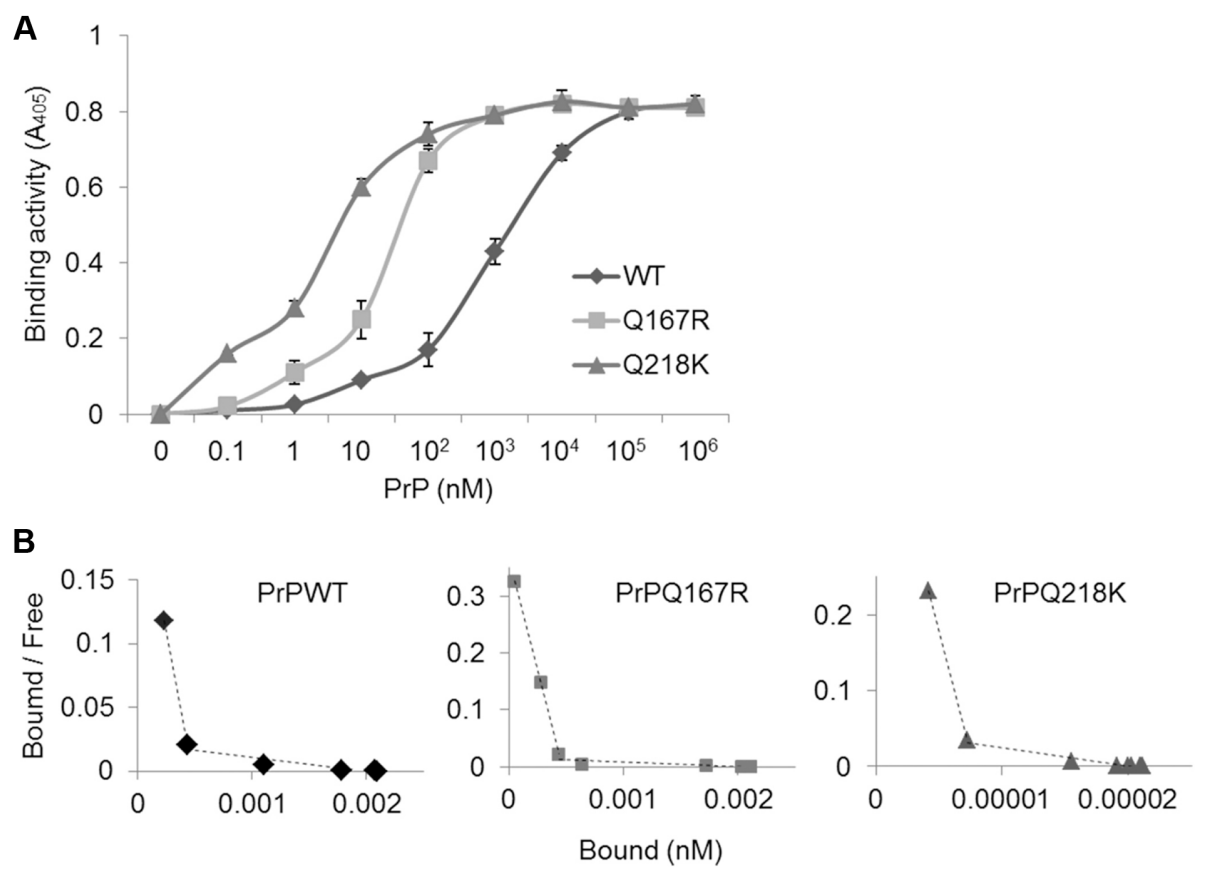

Fig. 4. Protein-protein interaction of wild-type (WT) and dominant negative (DN) mutant prion proteins (PrPs) with kringle domains of plasminogen.

(A) Relative binding activity of PrPWT, PrPQ167R, and PrPQ218K to immobilized kringle domains. Captured PrP molecules were assayed by antibody-mediated colorimetric detection at $A_{405}$. (B) Scatchard plots. The data in panel A were transformed using the Scatchard equation. The two different dotted lines indicate two separate binding sites for interaction.

\section{Competitive Binding Studies}

With a focus on binding specificity contributed by altered residues of DN mutant PrPs in the interaction with kringle domains of plasminogen, competitive binding tests were performed. Because Q167 and Q218 residues were changed into $\mathrm{R}$ and $\mathrm{K}$ residues, respectively, in DN mutant PrPs, which enhanced the binding affinity to kringle domains, the effect of free L-lysine and L-arginine on the interaction was examined. The competition was dependent on the concentration of L-lysine or L-arginine for all PrPs (Figs. 5A and 5B). L-Lysine was more efficient in interfering with the binding of kringle domains to PrP218K than to PrPWT and PrPQ167R (Fig. 5A). L-Arginine showed similar interference: the interaction of kringle domains with PrPQ167R was more efficiently blocked than with PrPWT or PrPQ218K (Fig. 5B). However, a negatively charged amino acid, L-aspartic acid, and a neutral amino acid, L-alanine, were unable to influence the interaction (Figs. 5C and 5D). These results indicate that both arginine at the 167th and lysine at the 218th amino acid residues are critical in the interaction between DN mutant PrPs and kringle domains.

\section{Discussion}

In the current study, we report the expression and purification of two DN mutant PrPs (PrPQ167R and PrPQ218K), their conformational properties, and interaction with kringle domains of plasminogen. Although the interaction of WT PrP and PrP218K with plasminogen was previously described [16], biochemical aspects of the interaction between PrPQ167R and plasminogen, particularly kringle domains, are discussed for the first time in this study. Together with that of PrPQ218K, the biochemical characteristic of PrPQ167R is notable when it interacts with kringle domains. Among four amino acid residues (Q167, Q171, V214, and Q218) of PrP previously proposed to interact with the hypothetical cofactor (Fig. 1), substitutions at the residues Q167 and Q218 were found as naturally occurring polymorphisms, which had profound influence in determining resistance to prion diseases. In sheep scrapie, PrP with Q171, which corresponds to Q167 in mouse PrP, directed high susceptibility to disease, whereas the $\operatorname{PrP}$ variant with R171 led to resistance [27-32]. In CreutzfeldtJakob disease of humans, PrP with K219 polymorphism, 
A

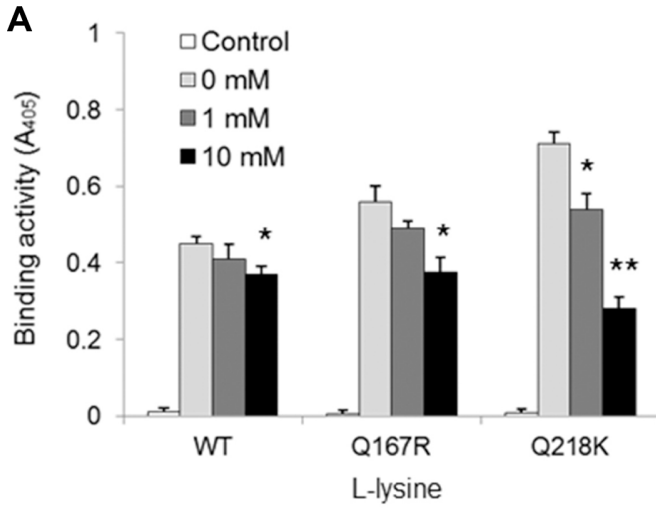

C

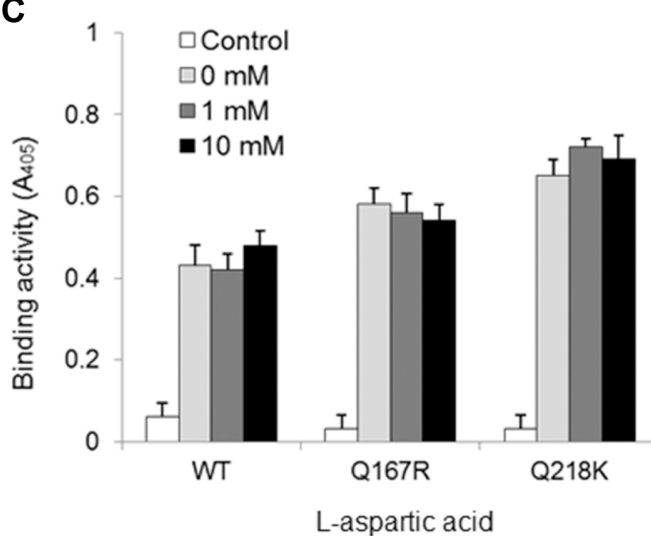

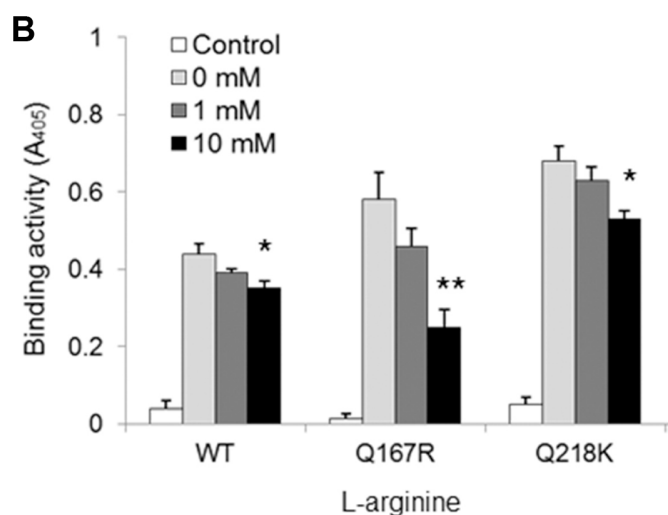

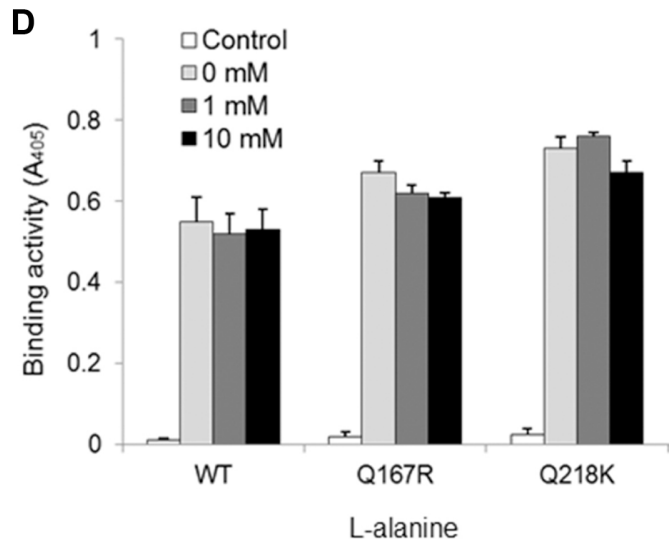

Fig. 5. Interference of interaction between dominant negative $(\mathrm{DN})$ mutant prion proteins (PrPs) and kringle domains of plasminogen.

Free amino acid competitors, L-lysine (A), L-arginine (B), L-aspartic acid (C), and L-alanine (D), were added at different final concentrations (0 (light grey bar), 1 (dark grey bar), and $10 \mathrm{mM}$ (filled bar)). No addition of recombinant PrP was used as the control (empty bar). Statistically significant differences of competitor activity were assessed in comparison with the reaction without competitor $(0 \mathrm{mM})\left(n>3 ;{ }^{*}, p<0.05 ;{ }^{* *}, p\right.$ $<0.01)$. No asterisks indicate "not statistical significant."

which corresponds to $\mathrm{K} 218$ in mouse $\mathrm{PrP}$, appeared to protect from disease [33, 34]. Thus, investigation on polymorphic variants of PrP, like PrPQ167R and PrPQ218K, is biologically more relevant than that on PrP variants of other polymorphisms.

This study revealed that the binding affinity of PrPQ167R to kringle domains was greater than that of WT PrP, but lower than that of PrPQ218K (Fig. 4). This suggests that, although the Q167 residue plays an important role, the Q218 residue could be more critical than Q167 in the hypothetical role of plasminogen during the conversion of $\operatorname{PrP}^{\mathrm{C}}$ to $\mathrm{PrP}^{\mathrm{Sc}}$. In this context, similar investigation using PrPQ167R/Q218K double DN mutants remains to be conducted for deciphering the contribution of each residue in the interaction with plasminogen.

In particular, the current study provides biochemical evidence for the interaction of $\mathrm{DN}$ mutant $\operatorname{PrP}$ with a putative cofactor, plasminogen. The mode of this interaction supports the predicted mechanism of DN inhibition of $\mathrm{Pr}^{\mathrm{SC}}$ propagation. For prion biosynthesis and propagation, although the precise mechanism is still unclear and much remains to be investigated, self-perpetuating replication of the $\operatorname{PrP}^{\mathrm{Sc}}$ based on the template-assisted prion conversion model with auxiliary cofactor(s) has been widely accepted [1]. In addition, it is hypothesized that DN mutant PrPs are less susceptible to be converted into $\operatorname{PrP}^{\mathrm{Sc}}$, presumably due to the stronger binding affinity between DN mutant PrPs and cofactor than WT PrP $[6,11,16,19]$.

A series of experimental findings suggest that plasminogen interacts with $\operatorname{PrP}$ and stimulates $\operatorname{PrP}^{S c}$ propagation in a concentration-dependent manner by accelerating the rate of $\operatorname{PrP}^{\mathrm{Sc}}$ generation, whereas depletion of plasminogen, destabilization of its structure, and interference with the PrP-plasminogen interaction hinder $\operatorname{PrP}^{\mathrm{Sc}}$ propagation in 
vitro $[9,14]$. Separately, previous reports revealed a number of detailed characteristics of the PrP interaction with kringle domains of plasminogen that serve as binding domains when plasminogen interacts with other proteins $[15,16]$. Among many others, the following are the two most relevant properties: first, PrP interacts with kringle domains in a cooperative manner, suggesting the presence of multiple binding sites in the kringle domains; and second, the binding of PrP with a mutation (PrPQ218K) to kringle domains is stronger than that of WT PrP.

Independently, previous studies have reported that point mutations at the 167th, 171st, 214th, or 218th residues of $\operatorname{PrP}^{\mathrm{C}}$ resulted in the $\mathrm{DN}$ effects on $\operatorname{PrP}^{\mathrm{Sc}}$ formation. Overexpression of these mutant $\operatorname{PrP}^{\mathrm{C}}$ molecules inhibited production of $\mathrm{PrP}^{\mathrm{Sc}}$ in prion-infected neuroblastoma cells in which endogenous WT PrP was expressed [6]. Similarly, in vivo experiments using transgenic animals expressing $\operatorname{PrP}^{\mathrm{C}}$ with either the Q167R or Q218K mutations confirmed and extended these results [19]. When infected with prions, transgenic mice expressing DN mutant $\operatorname{PrP}^{\mathrm{C}}$ under WT background transformation and showed delayed disease development and decreased $\operatorname{Pr}^{P^{S c}}$ accumulation. Furthermore, prion-infected transgenic mice expressing $\mathrm{DN}$ mutant $\operatorname{PrP}^{\mathrm{C}}$ on the PrP gene-null background showed no clinical or histopathological abnormalities and no $\operatorname{PrP}^{\mathrm{Sc}_{\mathrm{c}}}$ formation. A strain-dependent DN effect was also shown in transgenic mice overexpressing PrPQ171S [35].

Therefore, investigation to confirm whether plasminogen interaction with PrPQ167R follows the previously reported binding characteristics is critical in filling the gap between the functional and binding results available so far. In accordance with the characteristics known for the interaction of kringle domains with either WT PrP or PrPQ218K, the interaction of kringle domains with PrPQ167R resembles the binding properties, such as positive cooperativity in binding to kringle domains and a higher binding affinity than WT PrP. Taken together, the current study describing the interaction of PrPQ167R with plasminogen supports the DN effect in cultured cells and transgenic studies expressing the DN mutant PrPs. It also proposes that plasminogen shares a common characteristic with the previously hypothesized protein cofactor.

\section{Acknowledgments}

This work was supported by a research fund from Hanyang University (HUY-2012-G). The Authors thank Dr. H-J Woo (Seoul National University, Korea) for providing the goat anti-PrP antisera.

\section{References}

1. Prusiner SB. 1998. Prions. Proc. Natl. Acad. Sci. USA 95: 13363-13383.

2. Prusiner SB. 1991. Molecular biology of prion diseases. Science 252: 1515-1522.

3. Rogers M, Yehiely F, Scott M, Prusiner SB. 1993. Conversion of truncated and elongated prion proteins into the scrapie isoform in cultured cells. Proc. Natl. Acad. Sci. USA 90: 31823186.

4. Cohen FE, Pan K-M, Huang Z, Baldwin M, Fletterick RJ, Prusiner SB. 1994. Structural clues to prion replication. Science 264: 530-531.

5. Telling GC, Scott M, Mastrianni J, Gabizon R, Torchia M, Cohen FE, et al. 1995. Prion propagation in mice expressing human and chimeric PrP transgenes implicates the interaction of cellular PrP with another protein. Cell 83: 79-90.

6. Kaneko K, Zulianello L, Scott M, Cooper CM, Wallace AC, James TL, et al. 1997. Evidence for protein X binding to a discontinuous epitope on the cellular prion protein during scrapie prion propagation. Proc. Natl. Acad. Sci. USA 94: 10069-10074.

7. Peretz D, Williamson RA, Matsunaga Y, Serban H, Pinilla C, Bastidas RB, et al. 1997. A conformational transition at the $\mathrm{N}$-terminus of the prion protein features in formation of the scrapie isoform. J. Mol. Biol. 273: 614-622.

8. Zulianello L, Kaneko K, Scott M, Erpel S, Han D, Cohen FE, et al. 2000. Dominant-negative inhibition of prion formation diminished by deletion mutagenesis of the prion protein. $J$. Virol. 74: 4351-4360.

9. Mays CE, Ryou C. 2011. Plasminogen: a cellular protein cofactor for $\mathrm{PrP}^{\mathrm{Sc}}$ propagation. Prion 5: 22-27.

10. Cohen FE, Prusiner SB. 1998. Pathologic conformations of prion proteins. Annu. Rev. Biochem. 67: 793-819.

11. Perrier V, Wallace AC, Kaneko K, Safar J, Prusiner SB, Cohen FE. 2000. Mimicking dominant negative inhibition of prion replication through structure-based drug design. Proc. Natl. Acad. Sci. USA 97: 6073-6078.

12. Deleault NR, Piro JR, Walsh DJ, Wang F, Ma J, Geoghegan JC, et al. 2012. Isolation of phosphatidylethanolamine as a solitary cofactor for prion formation in the absence of nucleic acids. Proc. Natl. Acad. Sci. USA 109: 8546-8551.

13. Ryou C. 2007. Prions and prion diseases: fundamentals and mechanistic details. J. Microbiol. Biotechnol. 17: 1059-1070.

14. Mays CE, Ryou C. 2010. Plasminogen stimulates propagation of protease-resistant prion protein in vitro. FASEB J. 24: 5102-5112.

15. Fischer MB, Roeckl C, Parizek P, Schwarz HP, Aguzzi A. 2000. Binding of disease-associated prion protein to plasminogen. Nature 408: 479-483.

16. Ryou C, Prusiner SB, Legname G. 2003. Cooperative binding of dominant-negative prion protein to kringle domains. J. Mol. Biol. 329: 323-333. 
17. Maissen M, Roeckl C, Glatzel M, Goldmann W, Aguzzi A 2001. Plasminogen binds to disease-associated prion protein of multiple species. Lancet 357: 2026-2028.

18. Negredo C, Monks E, Sweeney T. 2007. A novel real-time ultrasonic method for prion protein detection using plasminogen as a capture molecule. BMC Biotechnol. 7: 43.

19. Perrier V, Kaneko K, Safar J, Vergara J, Tremblay P, DeArmond SJ, et al. 2002. Dominant-negative inhibition of prion replication in transgenic mice. Proc. Natl. Acad. Sci. USA 99: 13079-13084.

20. Shaked Y, Engelstein R, Gabizon R. 2002. The binding of prion proteins to serum components is affected by detergent extraction conditions. J. Neurochem. 82: 1-5.

21. Shin W, Lee B, Hong S, Ryou C, Kwon M. 2008. Cloning and expression of a prion protein $(\operatorname{PrP})$ gene from Korean bovine (Bos taurus coreanae) and production of rabbit antibovine PrP antibody. Biotechnol. Lett. 30: 1705-1711.

22. Warrens AN, Jones MD, Lechler RI. 1997. Splicing by overlap extension by PCR using asymmetric amplification: an improved technique for the generation of hybrid proteins of immunological interest. Gene 186: 29-35.

23. Mehlhorn I, Groth D, Stockel J, Moffat B, Reilly D, Yansura D, et al. 1996. High-level expression and characterization of a purified 142-residue polypeptide of the prion protein. Biochemistry 35: 5528-5537.

24. Kuwajima K. 1995. Circular dichroism, pp. 115-135. In Shirley BA (ed.). Methods in Molecular Biology: Protein Stability and Folding: Theory and Practice. Humana Press, Totowa. USA.

25. Choi BR, Lee J, Kim SY, Yim I, Kim EH, Woo HJ. 2013. Prion protein conversion induced by trivalent iron in vesicular trafficking. Biochem. Biophys. Res. Commun. 432: 539-544.

26. Scatchard G. 1949. The attractions of proteins for small molecules and ions. Ann. N. Y. Acad. Sci. 51: 660-672.

27. Goldmann W, Hunter N, Smith G, Foster J, Hope J. 1994. PrP genotype and agent effects in scrapie: change in allelic interaction with different isolates of agent in sheep, a natural host of scrapie. J. Gen. Virol. 75: 989-995.

28. Westaway D, Zuliani V, Cooper CM, Da Costa M, Neuman S, Jenny AL, et al. 1994. Homozygosity for prion protein alleles encoding glutamine-171 renders sheep susceptible to natural scrapie. Genes Dev. 8: 959-969.

29. Clousard C, Beaudry P, Elsen JM, Milan D, Dussaucy M, Bounneau C, et al. 1995. Different allelic effects of the codons 136 and 171 of the prion protein gene in sheep with natural scrapie. J. Gen. Virol. 76: 2097-2101.

30. Bossers A, Schreuder BE, Muileman IH, Belt PB, Smits MA. 1996. PrP genotype contributes to determining survival times of sheep with natural scrapie. J. Gen. Virol. 77: 2669-2673.

31. Hunter N, Moore L, Hosie BD, Dingwall WS, Greig A. 1997. Association between natural scrapie and PrP genotype in a flock of Suffolk sheep in Scotland. Vet. Rec. 140: 59-63.

32. Belt PB, Muileman IH, Schreuder BEC, Ruijter JB, Gielkens ALJ, Smits MA. 1995. Identification of five allelic variants of the sheep $\operatorname{PrP}$ gene and their association with natural scrapie. J. Gen. Virol. 76: 509-517.

33. Shibuya S, Higuchi J, Shin R-W, Tateishi J, Kitamoto T. 1998. Protective prion protein polymorphisms against sporadic Creutzfeldt-Jakob disease. Lancet 351: 419.

34. Shibuya S, Higuchi J, Shin R-W, Tateishi J, Kitamoto T. 1998. Codon 219 Lys allele of PRNP is not found in sporadic Creutzfeldt-Jakob disease. Ann. Neurol. 43: 826-828.

35. Striebel JF, Race B, Meade-White KD, LaCasse R, Chesebro B. 2011. Strain specific resistance to murine scrapie associated with a naturally occurring human prion protein polymorphism at residue 171. PLoS Pathog. 7: e1002275. 